Vol. 19(1):46-55, abril - julio 2016

\title{
El transitar de la muerte: cuidado paliativo ¿utopía o realidad?
}

\author{
The wandering of death: Palliative care, utopia or reality? \\ O trafegar da morte: cuidados paliativos, utopia ou realidade?
}

Nathaly Rozo-Gutiérrez, Enf., MSc. *

\begin{abstract}
Resumen
Introducción: El incremento de la esperanza de vida se duplicó en un siglo, y la dinámica de los perfiles de morbimortalidad tomó un giro hacia el predominio de las enfermedades no transmisibles. Las condiciones crónicas y progresivas alteran todas las dimensiones del ser y conllevan el deterioro paulatino del individuo, lo que se traduce en un incremento de la necesidad de cuidado. El cuidado surge como soporte integral para lograr el más alto nivel de confort y disminuir el impacto de la enfermedad en el paciente y su familia. Objetivo: Este manuscrito tiene el propósito de describir las realidades, avances y desafíos del cuidado paliativo (CP) para los actores del sistema de salud en Colombia. Resultados: El incremento de personas en el mundo que requieren de cuidado paliativo impulsa a organismos internacionales a orientar los países frente a la integración del cuidado paliativo en los sistemas de salud, con énfasis en la atención primaria en salud, comunitaria y domiciliaria. El mundo reporta una dinámica heterogénea del cuidado paliativo, mientras que los países desarrollados aumentan el acceso, cobertura y la atención multinivel con énfasis en el ámbito comunitario. Países de América Latina y el Caribe se concentran en la atención desde una provisión aislada del cuidado paliativo del sistema de salud. Conclusiones: El desarrollo del cuidado paliativo implica no sólo la construcción de políticas que reconozcan la atención paliativa, sino que también requiere sistemas de apoyo desde todos los niveles de atención, fortalecimiento de las
\end{abstract}

acciones comunitarias, desarrollo de habilidades en los profesionales de la salud junto con la integración de los servicios de salud para lograr el acceso y cobertura universal. [Rozo-Gutiérrez N. El transitar de la muerte: cuidado paliativo ¿utopía o realidad?. MedUNAB 2016; 19(1):46-55]

Palabras clave: Cuidados Paliativos; Cuidados Paliativos al Final de la Vida; Muerte; Calidad de Vida; Espiritualidad.

\begin{abstract}
Introduction: The increase in life expectancy has doubled in a century, and the dynamics of morbimortality profiles took a turn to the predominance of non-communicable diseases. Chronic and progressive conditions alter all dimensions of the human beings and lead to a progressive deterioration of the individual, which results in the increase of the need for care. The care emerges as comprehensive support to achieve the highest level of comfort and to decrease the impact of the disease on the patient and his/her family. Objective: This manuscript is intended to describe the realities, achievements and challenges of palliative care (PC) for the actors of the Colombian health system. Results: The worldwide growth of people who require palliative care encourages international organizations to guide countries towards the integration of palliative care into health systems, emphasizing on primary, community, and home health care.
\end{abstract}

\footnotetext{
* Enfermera, Magister en Salud Pública, Facultad de Medicina, Universidad El Bosque, Colombia.
} 
The world reports a heterogeneous dynamic of palliative care, while developed countries increase access, coverage and a multi-level care with an emphasis on the community level; Latin America and the Caribbean countries focus on the attention from an isolated supply of palliative care of the health system. Conclusions: The development of palliative care involves not only the creation of policies that recognize palliative care, but also requires support systems from all levels of care, strengthening of community actions, developing skills in health professionals along with the integration of health services to achieve access and coverage around the world. [Rozo-Gutiérrez N. The wandering of death: Palliative care, utopia or reality?. MedUNAB 2016; 19(1):46-55]

Key words: Palliative Care; Hospice Care; Death; Quality of Life; Spirituality.

\section{Resumo}

Introdução: O aumento da expectativa de vida dobrou-se num século, e a dinâmica dos perfis de morbidade e mortalidade virou-se para dar uma predominância de doenças não transmissíveis. As doenças crônicas e progressivas, afetam e deterioram o ser do indivíduo, levando-o à sentir necessidade de cuidados. O cuidado

\section{Introducción}

Las enfermedades no transmisibles (ENT) constituyen un importante problema de salud pública al ocupar las primeras causas de morbilidad, discapacidad y muerte $(1,2)$. Las ENT generan deterioro progresivo del individuo (3) en términos de funcionalidad física, cognitiva y emocional con repercusiones en su calidad de vida $(4,5)$; el avance tecnológico y de la atención en salud, así como las transiciones demográficas de los países han reportado un incremento del envejecimiento con mayor probabilidad de aumento de enfermedades crónicas (6). Para América Latina y el Caribe estas enfermedades han incrementado del $59 \%$ al $62.6 \%$ en menos de quince años (7).

El incremento de la esperanza de vida se duplicó en un siglo, y la dinámica de los perfiles de morbimortalidad tomó un giro hacia el predominio de las enfermedades no transmisibles (8), especialmente en los países de ingresos bajos y medios (3). La Organización Mundial de la Salud muestra que en los últimos años se han definido cerca de doce tipos de enfermedades crónicas y degenerativas, que requieren atención continua (9), lo que representa una alta cantidad de gastos en salud (10); siendo un desafío para la atención sanitaria al final de la vida, especialmente en aquellos grupos con mayor edad (8).

Las condiciones crónicas y progresivas producen vulnerabilidad a quien las padece, porque alteran todas las dimensiones del ser, desde la estructura corporal externa hasta la interna, comprometiendo de forma valiosa el mundo afectivo y relacional (11), lo que incrementa la percepción de emerge como suporte abrangente para atingir o mais alto nível de conforto e diminuir o impacto da doença no paciente e na sua família. Objetivo: Este manuscrito visa a descrever as realidades, conquistas e desafios dos cuidados paliativos (CP) para os atores do sistema de saúde na Colômbia. Resultados: O aumento de pessoas no mundo que necessitam de cuidados paliativos encoraja às organizações internacionais para que os países integrem os cuidados paliativos nos sistemas de saúde, como parte dos cuidados básicos de saúde, na comunidade e em casa. No mundo existe uma dinâmica heterogênea dos cuidados paliativos, enquanto nos países desenvolvidos aumenta o acesso e atenção em diversos níveis, com ênfase no estilo comunitário, na América Latina e o Caribe a atenção e o cuidado paliativo raramente se aplica e se faz de maneira não integrada ao sistema de saúde. Conclusões: Aevolução dos cuidados paliativos envolve a construção de políticas que reconheçam os cuidados paliativos e requer dos sistemas de apoio em todos os níveis de atenção, o reforço da ação da comunidade, desenvolvendo habilidades nos profissionais de saúde, juntamente com a integração dos serviços de saúde para alcançar o acesso universal e a cobertura. [RozoGutiérrez N. O trafegar da morte: cuidados paliativos, utopia ou realidade?. MedUNAB 2016; 19(1):46-55]

Palavras-chave: Cuidados Paliativos; Cuidados Paliativos na Terminalidade da Vida; Morte; Qualidade de Vida; Espiritualidade.

la "falta de futuro, amenazas a la destrucción física, social y psicológica, pérdida de los roles que hasta el momento ha desempeñado, disminución de la autoestima, sensación de ser carga para los demás, preocupaciones sobre la vida y la muerte, pensamientos negativos, falta de compañía así como aislamiento familiar y social" (12). Lo anterior se extiende hasta la esfera familiar por los cambios relacionales que produce, ya que interrumpe la cotidianidad social, laboral y económica de la misma (13).

Se estima que en el mundo unos 40 millones de personas tienen necesidades de cuidado paliativo, lo que proyecta la magnitud de la prestación de los servicios (12) y la demanda de cuidados formales e informales que se requieren $(4,14)$, junto a los recursos necesarios para su garantía. Es necesario que el cuidado paliativo sea visto como un soporte integral para lograr el más alto nivel de confort y disminuir el impacto de la enfermedad en el paciente y su familia (13).

De esta manera, el Cuidado Paliativo se centra en la prestación de la atención emocional y espiritual, junto a la gestión del dolor físico con la intención de satisfacer las necesidades y evitar el ejercicio de acciones terapéuticas innecesarias que prolonguen la agonía. Se facilita la aceptación de los cambios, sin intervenir ni influir en la decisión del paciente y su familia (15), teniendo en cuenta el marco de muerte digna y del ejercicio de la libertad para la toma de decisiones relacionadas con procedimientos y tratamientos para la prolongación de la vida $(4,16)$; de igual forma posibilita el desarrollo de herramientas para la identificación temprana de pacientes susceptibles de beneficiarse de los cuidados paliativos (17). 
Además, despliega intervenciones desde la prevención, alivio, evaluación continua e intervención bajo el enfoque de la totalidad del ser humano: autonomía, justicia y beneficencia, los cuales garantizan la verdad, comunicación, confianza, satisfacción y confort (12); estos cuidados inician desde el momento del diagnóstico, se agudizan días previos a la muerte y finalizan con el acompañamiento a la familia en relación con la atención del duelo (18).

El desarrollo del cuidado paliativo en el mundo está relacionado con el avance de políticas que integran el cuidado paliativo al sistema de salud, dentro de un marco de necesidad de atención, oferta de servicios integrales e integrados, así como asignación de recursos para el acceso y cobertura $(19,20)$.

Un estudio realizado a nivel mundial sobre la calidad de la atención a las personas cuyo pronóstico de vida es reservado y están próximas a morir, identificó que países como Reino Unido, Australia, Nueva Zelanda reportan un índice de calidad de la muerte significativamente mayor en comparación con el resto del mundo (21).

Otros estudios desarrollados en países como España y Estados Unidos han evidenciado que aproximadamente entre el $46 \%$ y el $72.1 \%$ de la totalidad de pacientes con enfermedades no transmisibles al ser valorados no contaban con cuidado paliativo $(22,23)$. Los países en vía de desarrollo que constituyen el $80 \%$ de la población global, calculan que sólo el 6\% accede al consumo de morfina (3); siendo indicadores mundiales de la vigilancia integral para la prevención y control de las enfermedades no transmisibles.

Es por esto que el cuidado paliativo requiere un abordaje desde la salud pública que posibilite el desarrollo de políticas integrativas hacia la salud universal en el cuidado paliativo (23); teniendo en cuenta que es un área de la atención sanitaria objeto de preocupación y reflexión por parte de organismos internacionales y nacionales, dado que exige enormes esfuerzos y retos para los Gobiernos, los Sistemas de Salud, los profesionales de salud y la sociedad misma (24). Por eso, es necesaria la escritura de artículos de reflexión que contribuyan al debate en torno al cuidado paliativo en el mundo y en Colombia. Este manuscrito tiene el propósito de describir las realidades, avances y desafíos del Cuidado paliativo para los actores del sistema de salud en Colombia.

\section{Resultados}

\section{Suceso vital: Experiencia vivida.}

Paciente de 96 años de edad, hospitalizada en una Institución de Salud, trasladada a Unidad de Cuidado Intensivo por deterioro de su condición de salud, asociada a múltiples comorbilidades: colelitiasis, nefropatía, insuficiencia cardíaca congestiva, con posibilidad de realizar de primera mano opciones terapéuticas curativas. Sin embargo, los familiares toman la decisión de rechazar la conducta terapéutica curativa de tipo quirúrgico, con la posibilidad de continuar la atención meramente paliativa: analgésicos, opiáceos, oxígeno, sedantes, entre otros.

A pesar de que los familiares expresan por medio escrito que reconocen la gravedad de la condición patológica de la paciente, consideran innecesaria la realización de medidas terapéuticas orientadas al sostén de la vida, tales como la reanimación, porque para ellos prima la calidad de vida de la persona que sufre sobre el potencial terapéutico. Ante esto, se solicitó claramente la limitación del esfuerzo terapéutico, diagnóstico y de reanimación, para así disminuir la prolongación del dolor del paciente y el sufrimiento emocional de sus familiares.

Sin embargo, la solicitud expresada por los familiares de manera clara, soportada por marcos normativos, no fue tenida en cuenta por varios profesionales de la salud, quienes concibieron el rechazo de medidas invasivas como acciones terapéuticas de abandono y de facilitar la muerte en condiciones no seguras para el paciente.

Lo anterior causó procesos administrativos inaceptables como información no clara del proceso de atención realizado, ausencia de autorizaciones para solicitar apoyo domiciliario y cuidado en casa, inadecuada atención biopsicosocial para el paciente y su familia, no formulación de medicamentos para el manejo del dolor sumados a actitudes agresivas, amenazantes para abandonar la Institución. Además, se negaron los medios para disminuir el sufrimiento y el dolor de la paciente y su familia.

La decisión familiar por el cuidado paliativo debió ser solicitada empleando medios legales. A partir de este proceso se obtuvo una atención humana, oportuna y paliativa en los períodos que antecedieron a la muerte. La paciente murió en un ambiente donde el dolor físico fue controlado, con soporte médico, y junto a sus familiares en condiciones dignas. Es claro que la aceptación de la muerte habría sido más dolorosa para ambas partes si no hubiera existido una serie de soportes paliativos dignos para que el proceso de la muerte, con unos marcos de cumplimiento de derechos y deberes institucionales, se consolidara previo al deceso.

\section{¿Cómo ha sido la concepción de la muerte a través de la historia?}

El concepto de la muerte "tiene el mismo origen que la cultura" (25), por tanto, es derivado de construcciones sociales que pueden transitar desde abordajes mágicoreligiosos hasta político-económicos. Algunos han tenido miradas reduccionistas: la muerte es un hecho biológico de observación a través del cese de los latidos cardiacos $(25$, 26). Otros, han utilizado su poder catastrófico como instrumento simbólico de control y dominación de individuos y colectivos. En contraposición, la muerte para muchos otorga poder y sabiduría (21). 
A la fecha existen infinidades de conceptos, miradas y abordajes en torno a la muerte; a pesar de ello, todos coinciden en que su comportamiento es complejo e incierto. Es un generador de caos e incertidumbre; todo se desconoce sobre la muerte, el acercamiento más próximo es a la actitud que se tiene sobre ella, derivado de sensaciones de dolor, agonía, sensaciones de pérdida de control, dolor, desconcierto y total dependencia funcional (27); atribuido a la condición biológica que experimente el individuo (25).

La cercanía con la muerte produce diversas emociones, que en este caso, pueden transitar desde la sorpresa ante el diagnóstico del mal pronóstico, hasta la comprensión de la muerte como lo único cierto en la vida (28). Se convierte en la oportunidad para renacer y disfrutar la vida a plenitud, llenándose de posibilidades con sus seres queridos (29).

\section{¿Cuáles son las manifestaciones de negación de la muerte como suceso natural de la vida?}

La muerte, como un fenómeno de la realidad humana, que representa el final de la vida y le proporciona al ser humano la conciencia de finitud, ha estado sujeta a la transformación de la sociedad actual, inmersa en una escala de valores, símbolos e ideologías de toda índole que ha maximizado el temor por enfrentarla (25). El temor hacia los momentos de dolor físico y emocional que produce, así como a la pérdida de autonomía y dependencia funcional (30).

El avance tecnológico en la medicina ha fortalecido la detección, tratamiento y rehabilitación de las enfermedades, acrecentando la necesidad biológica de prolongar la vida. Dicha prolongación debe realizarse bajo parámetros bioéticos de beneficencia, no maleficencia, autonomía y justicia, donde la calidad de vida sea el parámetro para las decisiones éticas-médicas. El proceso de atención en salud debe contemplar el momento en que la muerte sea aceptada como un proceso natural de la vida, que posibilite el máximo cuidado hacia la muerte sin sufrimientos innecesarios (31).

Sin embargo, el desarrollo de corrientes vitalistas donde la prolongación de la vida de forma artificial es un proceso de lucha constante entre la tecnología y la biología, vulnera los derechos del paciente y su dignidad (31). La obstinación terapéutica es un reflejo de "encarnizamiento terapéutico" dado por "la agonía prolongada, el sufrimiento extremo, la desfiguración corporal y el aislamiento del paciente" (32), que implica el uso de múltiples recursos humanos y técnicos para hacer cada vez más invisible la frontera de la vida y de la muerte, sin tener en cuenta la calidad de vida que el paciente y su familia puedan tener durante el sometimiento terapéutico; convirtiéndose en una forma de cuidar artificialmente al cuerpo, y no al alma misma $(33,34)$.

\section{¿Cómo es la realidad del Cuidado Paliativo?}

Los últimos reportes estiman que cerca de 20 millones de personas en el mundo requieren cuidado paliativo (35), concentrándose en niños y en mayores de 60 años, especialmente en Europa, Sureste de Asia y Regiones de América (36). Lo anterior constituye un escenario para la prestación de servicios de salud orientados a brindar bienestar físico y emocional al paciente y su familia en ambientes hospitalarios y domiciliarios, ante la imposibilidad de la curación terapéutica $(28,37)$.

La Organización Mundial de la Salud, desde el año 2002 promulgó el nuevo enfoque de atención sanitaria de los países, basado en el alivio del dolor y acompañamiento para la elaboración del duelo (38). A partir de ello, en 2014 a través de la $67^{\mathrm{a}}$ Asamblea Mundial de la salud se solicita la formulación de políticas para el fortalecimiento del cuidado paliativo en los sistemas de salud con énfasis en la atención primaria en salud comunitaria y domiciliaria (39). Este marco legal permitió que los países diseñaran estrategias para el acceso universal a la salud, a través de políticas nacionales y planes de acción que posicionaran las enfermedades no transmisibles como prioridades en salud pública para el monitoreo, vigilancia, control y reorientación de los servicios de salud de acuerdo con las necesidades de los pacientes y sus familias (3).

El cuidado paliativo en el mundo ha tomado un enfoque integral con atención multidisciplinaria, que dispone un sistema de apoyo a la familia y complementa el tratamiento activo para las enfermedades (40), bajo la evidencia de que se disminuyen en un $13 \%$ los gastos en salud. Adicionalmente, el cuidado paliativo contribuye en la reducción de la prestación de los servicios de salud innecesarios, tales como los reingresos hospitalarios (41).

A la fecha se han hecho avances en el cuidado paliativo en Europa, Canadá, Australia y Estados Unidos donde se integra a la medicina convencional del sistema de salud; cuenta con el desarrollo de políticas de control y formulación de medicamentos para la disponibilidad de opioides, así como la asignación de recursos económicos, físicos y humanos para su diseño, desarrollo y evaluación (8).

El Atlas Europeo de Cuidados Paliativos reporta que existe un incremento en la disponibilidad de estos centros, transcurriendo de 86 registrados en el 2007 a 212 en el 2012 (42). De esta manera han fortalecido la gestión de los síntomas para lograr el confort y la dignidad humana al final de la vida (43).

América Latina y el Caribe reportan una dinámica heterogénea del cuidado paliativo. Chile, Costa Rica y Uruguay son países representativos de la región que cuentan con una integración preliminar a otros servicios del sistema de salud país, mientras que Argentina posee una dinámica generalizada del cuidado paliativo, sin la integración al sistema de salud. Por otro lado, Brasil, Colombia, Cuba, República Dominicana, Ecuador, El Salvador, Guatemala, México, Panamá, Paraguay, Perú y Venezuela muestran una provisión aislada de los servicios de cuidado paliativo (44).

Con respecto a los servicios de salud según los niveles de complejidad en el cuidado paliativo, se observa que en la 
región de Suramérica se concentra el mayor porcentaje en servicios hospitalarios, en comparación con la región de América del Norte donde predomina la atención desde el nivel comunitario (domicilio, hospicio y consulta en centro comunitario). De forma particular, Costa Rica y Chile son países que se caracterizan por el desarrollo de servicios multinivel para la población que requiere cuidado paliativo (44).

A pesar de los avances descritos en los países, los estudios desarrollados en torno al cuidado paliativo permiten identificar que entre el $51 \%$ y el $54 \%$ de los pacientes afirmaron preferir morir en casa, sin embargo, del $34 \%$ al $63 \%$ ocurren en un ambiente hospitalario (35), dado el predominio de este nivel de atención en los países descritos.

En relación con el cumplimiento de los indicadores de vigilancia y monitoreo sobre el avance en el cuidado paliativo, se observa con respecto al uso de opioides el incremento en el consumo, con mayor frecuencia en Argentina, Brasil, Chile y Costa Rica (44).

Es importante tener en cuenta que el cuidado paliativo en todos los niveles de atención, debe estar soportado desde programas nacionales de atención paliativa, que no sólo estén orientados hacia el manejo del dolor, sino también al abordaje de los aspectos psicológicos y espirituales que disminuyan el impacto social(13).

\section{¿Cuáles son las experiencias del Cuidado Paliativo?}

El cuidado paliativo es una prioridad transversal de las políticas de salud global (44), y sus avances han permitido posicionarlo en las agendas de gobierno de algunos países, como un tema trascendental para la calidad de vida de la población.

Un estudio desarrollado en Estados Unidos mostró que la implementación del cuidado paliativo y su integración con los servicios del sistema de salud redujeron la frecuencia de muertes intrahospitalarias del 2003 al 2012, así como las tasas de hospitalización en los últimos 12 meses. De igual forma se registró un aumento del uso de los servicios de cuidado paliativo del $29 \%$ al $42 \%$. Se ha confirmado, además, una reducción de los gastos atribuidos al cuidado de enfermedades no transmisibles por hospitalizaciones y reingresos (17). Otra investigación reporta que el cuidado paliativo mejora la calidad de vida del paciente y su familia, además de optimizar el tratamiento y prolongar la supervivencia (45).

Se ha observado que la capacidad de respuesta de los países de América Latina y el Caribe es escasa porque no satisface las demandas y los requerimientos del cuidado paliativo. Se ha calculado que solo entre el $5 \%$ y el $10 \%$ de pacientes acceden a este tipo de atención (46). De igual forma la evidencia reporta que el equipo de salud carece de las competencias para proporcionar cuidados al final de la vida, derivado de la formación centrada en la curación y evitación de la muerte (47).
Además, las evaluaciones clínicas durante el cuidado paliativo de los pacientes han facilitado el diagnóstico eficaz de las patologías y la detección temprana de complicaciones, que contribuyen a mejores resultados en el tratamiento, considerándose una estrategia costo-efectiva para el sistema de salud (37).

Por otro lado, los Programas consolidados en los últimos años han incluido al cuidador familiar, dentro de las intervenciones porque lo consideran un actor clave para la atención directa que posibilita la comprensión de las necesidades reales del paciente. Este abordaje permite que el cuidador tenga mayor aceptación de la muerte a través de la elaboración de un duelo anticipado: "movilizando emociones como la hostilidad, el deseo de muerte, la angustia y la culpa" (48), para dar paso a sentimientos positivos de afrontamiento que "posibilita la resignificación de la historia y del tiempo que queda por vivir" (48).

Los países con mayor disponibilidad de programas e intervenciones de cuidado paliativo son Reino Unido y España, sumado a Estados Unidos; la Asociación Internacional de Hospicios y Cuidado Paliativo (IAHPC) agrupa a cerca de 685 entidades registradas con este objeto social además de investigación y formación continua de profesionales. Para América Latina y el Caribe hay disponibles 95 Instituciones con sedes administrativas en México, Argentina, Brasil y Chile (21).

Así mismo se han construido grupos de discusión científica y académica alrededor del cuidado paliativo en todo el mundo a través de fundaciones y publicaciones periódicas con el propósito de compartir experiencias, dilemas, desarrollo de avances y evidencia a todos los países; entre estas organizaciones se encuentran: Fundación Europea de Cuidados Paliativos, la Sociedad Española de Cuidados Paliativos, la Revista Española de Medicina Paliativa, The British Medical Journal of Palliative Care, Guía Farmacológica en Cuidados Paliativos, Instituto de Cuidados Paliativos en la Universidad de Ottawa, The European Journal of Palliative Care, entre otras. En su mayoría financiadas por el sistema de salud, donaciones, organizaciones, aportes internacionales, eventos de caridad y contribuciones corporativas que se describen en la Tabla 1 $(18,40,49-52)$.

\section{¿Cuál es el panorama del Cuidado Paliativo en Colombia?}

Colombia es uno de los pioneros del cuidado paliativo en América Latina y el Caribe debido a la apertura de la primera Clínica del dolor y Cuidado paliativos de la Universidad de Antioquia en el año 1980 (44). Posteriormente, se introdujo el concepto de Cuidados Paliativos a través de la creación de una fundación, como el primer hospicio/centro de día. Para el año 1995 se consolidó el Instituto Nacional de Cancerología, como centro hospitalario especializado de referencia para el país que posibilita la formación universitaria y el desarrollo de estudios de investigación (44). 
Tabla 1. Grupos de científicos y académicos alrededor del cuidado paliativo en el mundo.

\begin{tabular}{|c|c|c|c|}
\hline CONTINENTE & PAís & DENOMINACIÓN & DESCRIPCIÓN \\
\hline \multirow[t]{6}{*}{ América Latina } & $\begin{array}{c}\text { México, Argentina, Brasil, } \\
\text { Colombia, Uruguay, } \\
\text { Chile, Costa Rica, } \\
\text { Honduras, República } \\
\text { Dominicana, Ecuador, El } \\
\text { Salvador, Guyana, } \\
\text { Jamaica, Nicaragua, } \\
\text { Panamá, Paraguay, } \\
\text { Perú, Puerto Rico y } \\
\text { Venezuela. }\end{array}$ & $\begin{array}{l}\text { Asociación de Cuidados } \\
\text { Paliativos (ASOCUP). }\end{array}$ & $\begin{array}{c}\text {-Escuela del buen morir, desarrollo de programas de } \\
\text { intervención y de educación al paciente y las familias } \\
\text {-Escenario para la realización de estudios de } \\
\text { investigación sobre el dolor. } \\
\text {-Integrada por } 16 \text { países. }\end{array}$ \\
\hline & México. & $\begin{array}{l}\text { Observatorio Internacional } \\
\text { de Cuidados Paliativos. }\end{array}$ & $\begin{array}{c}\text {-Programas y Unidades de Atención desde las } \\
\text { Secretarias de Salud del país } \\
\text {-Inclusión de contenidos del cuidado paliativo en } \\
\text { currículos de formación universitaria para profesionales } \\
\text { de la salud. } \\
\text {-Evidencia desde el marco jurídico que respalda la } \\
\text { atención paliativa en el país: tratamiento integral al dolor, } \\
\text { atención integral y paliativa }(49,58) \text {. }\end{array}$ \\
\hline & Cuba. & $\begin{array}{l}\text { Programa Nacional de } \\
\text { Atención al Dolor y } \\
\text { Cuidados paliativos. }\end{array}$ & $\begin{array}{c}\text { Investigaciones realizadas en torno a la eficacia, que } \\
\text { facilita el monitoreo del programa para el mejoramiento } \\
\text { de la atención integral contando con una extensa red de } \\
\text { cuidadores familiares }(50,59) \text {. }\end{array}$ \\
\hline & Brasil. & $\begin{array}{l}\text { Serviço de Cuidados } \\
\text { Paliativos ao Serviço da } \\
\text { Dor do Hospital de } \\
\text { Clinicas de Porto Alegre e, } \\
\text { depois, no Rio de Janeiro, } \\
\text { Instituto Nacional do } \\
\text { Câncer, }\end{array}$ & $\begin{array}{l}\text {-Desarrollado desde los años } 80(60) \text {. } \\
\text {-Desde el } 2008 \text { Conselho Regional de Medicina do } \\
\text { Estado de São Paulo consolidó un Programa de cuidado } \\
\text { paliativo para el país, teniendo en cuenta las directrices } \\
\text { internacionales y las necesidades nacionales }(51,61) \text {. }\end{array}$ \\
\hline & Chile. & $\begin{array}{l}\text { Programa alivio del dolor y } \\
\text { cuidado paliativo, }\end{array}$ & $\begin{array}{l}\text {-Implementado desde el año } 1995 \text { y vigente a la fecha } \\
\text {-Brinda atención integral a individuos y familias } \\
\text {-avances investigativos a través de medios cuantitativos } \\
\text { para valorar el cuidado, los factores relacionados y su } \\
\text { repercusión en el proceso de morir para el sujeto y su } \\
\text { familia }(52,62) \text {. }\end{array}$ \\
\hline & Argentina. & $\begin{array}{l}\text { Programa Argentino de } \\
\text { Medicina Paliativa. } \\
\text { Fundación FEMEBA. }\end{array}$ & $\begin{array}{l}\text {-Constituyeron espacios de interacción con familiares y } \\
\text { amigos a los pacientes que se encuentran } \\
\text { hospitalizados, cuenta con apoyo interdisciplinario. } \\
\text {-Brindan cuidado en casa, educación comunitaria, } \\
\text { soporte científico y estudios de investigación, formación } \\
\text { del talento humano e innovación en la atención, de } \\
\text { acuerdo con las necesidades de los individuos }(18,40) \text {. }\end{array}$ \\
\hline \multirow[t]{2}{*}{$\begin{array}{l}\text { América del } \\
\text { Norte }\end{array}$} & Canadá. & $\begin{array}{l}\text { Unidad de Cuidados } \\
\text { Paliativos en el Royal } \\
\text { Victoria Hospital }\end{array}$ & $\begin{array}{l}\text { Modelo de programa de atención desde } 1973, \text { y se ha } \\
\text { extendido a más de } 60 \text { países del mundo. }\end{array}$ \\
\hline & Florida, Estados Unidos. & Hope Hospice. & $\begin{array}{l}\text { La provisión del cuidado es interdiscipinaria a través de } \\
\text { la asistencia diaria, los servicios de cuidado y la } \\
\text { eliminación de atención fragmentaria }(18,40) \text {. }\end{array}$ \\
\hline
\end{tabular}




\begin{tabular}{|c|c|c|c|}
\hline \multirow[t]{2}{*}{ Europa } & Reino Unido. & Giles Hospice & $\begin{array}{l}\text {-Fue desarrollado a partir de la experiencia dada en } 1960 \\
\text { a través del St. Christhopher Hospice en Londres, donde } \\
\text { se brindó conocimientos a los cuidadores y se alivió el } \\
\text { sufrimiento de los individuos con intervenciones } \\
\text { psicoemocionales (60) } \\
\text {-Son servicios prestados por voluntarios y profesionales } \\
\text { donde fortalecen los recursos internos del ser humano } \\
\text { para afrontar las crisis vitales y circunstanciales. } \\
\text {-Cuenta con International Observatory on End of Life } \\
\text { Care - IOELC -, escenario científico que apoya el } \\
\text { desarrollo de experiencias y avances del cuidado } \\
\text { paliativo en el mundo }(52,61) \text {. }\end{array}$ \\
\hline & Rumania. & Hospice Care & $\begin{array}{c}\text { Cuidado paliativo de adultos y niños, con un equipo } \\
\text { interdisciplinario, acceso a medicamentos con } \\
\text { estándares de calidad }(18,40) \text {. }\end{array}$ \\
\hline \multirow[t]{2}{*}{ Asia } & Kerala, India. & $\begin{array}{l}\text { Neighbourdhood Network } \\
\text { in Palliative Care }\end{array}$ & $\begin{array}{l}\text {-Este programa comunitario aborda las necesidades } \\
\text { reconocidas por la comunidad. } \\
\text {-Desde el año } 2000 \text { se incluyeron voluntariados, y } \\
\text { actualmente cuenta con soporte profesional bajo el } \\
\text { Modelo de Cuidado Primaria en Salud }(18,40) \text {. }\end{array}$ \\
\hline & Vietnam. & $\begin{array}{c}\text { Model Paliative Care } \\
\text { Program el cual trabaja con } \\
\text { el apoyo del Harvard } \\
\text { Medical School Center for } \\
\text { Paliative Care }\end{array}$ & $\begin{array}{l}\text { Para la revisión técnica y normativa que facilite el } \\
\text { desarrollo de normas que regulen el acceso a } \\
\text { medicamentos como opioides }(18,40) \text {. }\end{array}$ \\
\hline África & Tanzania. & $\begin{array}{c}\text { Continum of Care for } \\
\text { Persons }\end{array}$ & $\begin{array}{c}\text { Dirigido a personas con VIH/SIDA que capacitan a la } \\
\text { comunidad sobre el cuidado en casa, dando seguimiento } \\
\text { y asistencia técnica y de gestión en salud }(18,40) \text {. }\end{array}$ \\
\hline
\end{tabular}

Fuente: Elaboración propia del autor.

Los gastos en salud (\%) del PIB para Colombia a través del tiempo se han incrementado; sin embargo, su porcentaje de 7.20, (2014) es inferior con respecto al mundo, de 9.94 (2014) (53). Lo que representa una barrera de sostenibilidad para la garantía de desarrollo y operatividad de servicios de cuidado paliativo. Lo anterior se refleja en la disponibilidad de menos de 0.5 servicios de cuidado paliativo totales por millón de habitantes. De acuerdo con la estimación de Lynch y cols., 2012, Colombia se ubica en el grupo de países con provisión aislada de los servicios de cuidado paliativo (44).

Los servicios disponibles se han caracterizado principalmente en unidades hospitalarias de $3^{\circ}$ y $4^{\circ}$ nivel de atención (57\%), contándose con 13 Instituciones de salud ubicadas en cuatro ciudades del país: Bogotá, Medellín, Cali y Pasto (44); seguido por residencias de tipo hospicio $(17 \%)$, equipos multinivel $(13 \%)$, atención domiciliaria ( $9 \%$ ) y, por último, unidades en hospitales de $2^{\circ}(4 \%)$ (44). A la fecha no se cuenta con equipos de apoyo hospitalario multidisciplinarios especializados en cuidado paliativo, así como tampoco con centros de día (44).

Con respecto al uso de opioides en Colombia, para el año 2010 se reportó $6.7 \mathrm{mg} /$ habitantes, superando la media de
América Latina y el Caribe de $4.80 \mathrm{mg} / \mathrm{habitante}$. Los principales opioides usados son: fentanil, morfina, oxicodona, metadona y otras (44).

Por otro lado, el país cuenta desde 2014 con la ley nacional de cuidados paliativos denominada "CONSUELO DEVIS SAAVEDRA", donde se reconoce el derecho a la atención integral de "personas con enfermedades en fase terminal, crónicas, degenerativas e irreversibles, que pretende mejorar la calidad de vida" (54) con la opción de desistir de procedimientos extraordinarios y el alivio del dolor a través del acceso y disponibilidad de opioides, los cuales se incluyen en el Plan Obligatorio de Salud del Sistema General de Seguridad Social en Salud (44). Sin embargo, no está articulada con un programa nacional de cuidado paliativo que se integre al sistema de seguridad social en salud. De forma aislada se han desarrollado guías de tratamiento para el control de los síntomas frecuentes en el cuidado paliativo desde el Ministerio de Salud y Protección Social.

Los demás recursos disponibles son la Asociación Nacional de Cuidados Paliativos, y la oferta académica universitaria para el fortalecimiento del recurso humano en 
salud específico para esta área de cuidado, donde se describe que $3 / 57$ facultades de medicina cuentan con esta formación durante el pregrado, además del desarrollado de programas de posgrado (especialidades clínicas y maestrías) específicas para medicina e interdisciplinarias (44). A pesar del incremento en la oferta académica la disponibilidad de recursos humanos en salud entrenados en cuidado paliativo continúa siendo baja con relación a la población total (44), teniendo en cuenta que la formación académica es una prioridad para impulsar un servicio de calidad (55).

De esta forma es visible que los avances del cuidado Paliativo en Colombia han sido alrededor de políticas legislativas que obligan la inclusión del cuidado paliativo dentro de los Programas de salud pública del país con énfasis en la atención primaria en salud, junto con la disponibilidad de opioides en las Instituciones sanitarias, el cuidado del paciente y su familia, así como la implementación de programas educativos para profesionales y cuidadores (21, 56). Sin embargo, a pesar de los avances descritos el cuidado paliativo en Colombia es aislado y se concentra en el nivel hospitalario en algunas zonas específicas del país, debe extenderse no sólo hacia todos los ámbitos comunitarios sino a todas las ciudades del país. Los avances del país deben visionar el desarrollo de la atención multinivel para el logro del acceso y cobertura universal de cuidado paliativo en el país.

\section{¿Cuáles son las tendencias del Cuidado Paliativo?}

El mundo continúa desarrollando esfuerzos y metodologías para avanzar en la implementación del cuidado paliativo. Una de ellas, es el juego de herramientas construido con miras a mejorar el cuidado desde una visión holística y con los mínimos establecidos para su ejecución, siendo un modelo que impulsa el desarrollo en países de bajos recursos en salud. Estas herramientas están configuradas como ejes orientadores para ser implementado el cuidado paliativo en un plan nacional integrado a los servicios del sistema de salud. La caja de herramientas está simbolizada a través de un árbol (Figura 1), donde sus ramas posicionan al sujeto de cuidado en todos los niveles de atención: centro de día, cuidado ambulatorio, hospitalario con un equipo de cuidado paliativo, y sus raíces constituyen los ejes transversales del cuidado: físico, psicológico, espiritual y social(57).

Los aspectos físicos no sólo hacen referencia a lo biológico del ser humano, también integra la necesidad de gestión de infraestructuras para el soporte paliativo que se requiere. Lo psicológico tiene una mirada desde los equipos multidisciplinarios y grupos de apoyo que orientan al paciente y su familia para el afrontamiento de la enfermedad y sus necesidades. El componente espiritual incluye no solo la atención individual sino también el abordaje desde grupos, como principal herramienta para acentuar la esperanza o facilitar la aceptación del transitar de la vida hacia la muerte $(14,34,58)$.

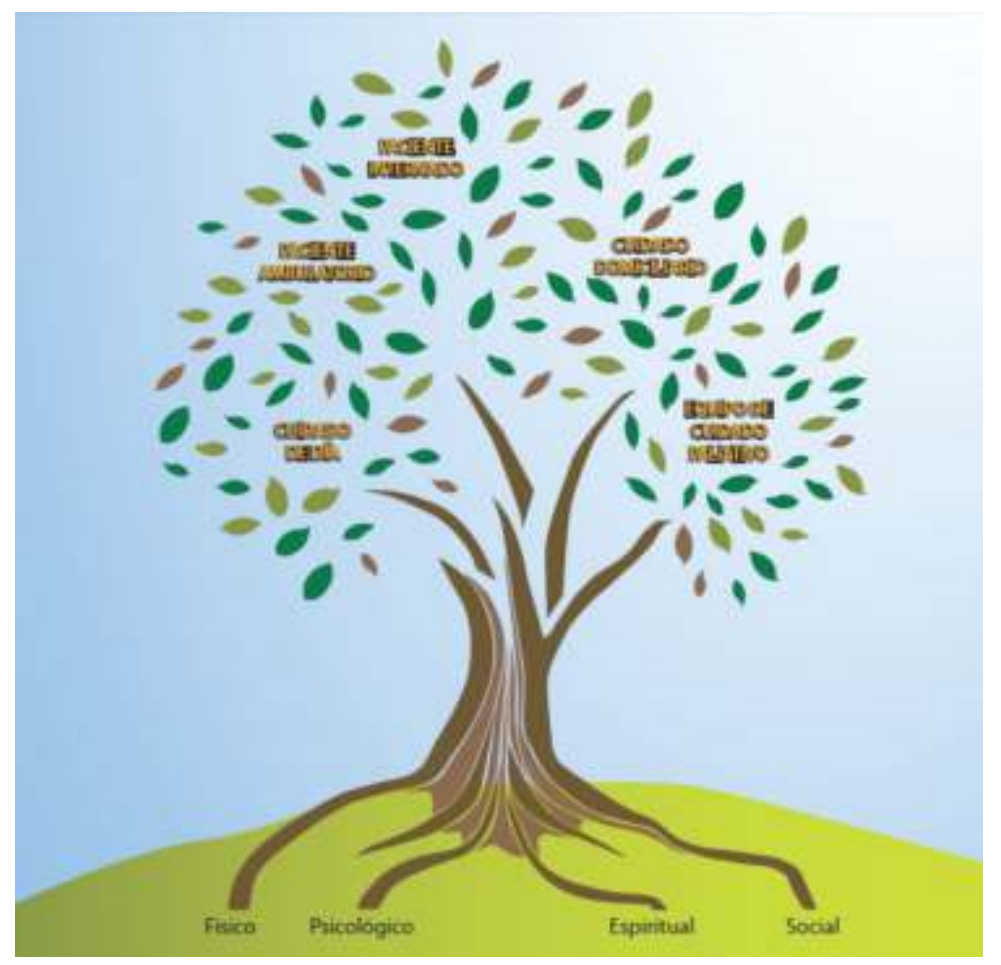

Fuente: Help the Hospices, Worldwide Palliative Care Alliance. Juego de herramientas para el Cuidado Paliativo: Mejorando el cuidado desde la raíz en sectores limitados en recursos. 2008. Londres-Reino Unido. ISBN: 978-1871978-71-1

Figura 1. Juego de herramientas para el cuidado paliativo. 
Y por último el componente social, aquel que integra las instituciones gubernamentales y no gubernamentales, los profesionales de salud y los recursos comunitarios, mediante un trabajo intersectorial e interinstitucional facilitan la prestación de los servicios, el acceso a los medicamentos, la gestión de recursos de financiación y la evaluación del cuidado paliativo a través de la reducción del impacto de la enfermedad en el paciente y su familia (59).

Es importante que todos los actores de salud comprendan que el cuidado paliativo es ilimitado y responde a las necesidades humanas, haciendo posible las modalidades del cuidado paliativo: hospitalarias, domiciliarias, ambulatorias, servicios de atención de emergencias y centro día (60). La inclusión de la tecnología en el cuidado de las personas, a través de los dispositivos móviles, debe ser una vanguardia del cuidado paliativo en el sistema de salud de los países, porque facilita la comunicación continua del paciente y su familia con el equipo de salud y/o los grupos de apoyo (45).

\section{Conclusiones}

El avance de la tecnología y el tratamiento de las enfermedades, sumando al aumento de la esperanza de vida en los países, conlleva al incremento de la longevidad y de las enfermedades crónicas. Siendo cambios demográficos que contrarrestan desafíos para los sistemas de salud, desplazando la atención hospitalaria y curativa hacia una atención multinivel: comunitaria, domiciliaria y ambulatoria desde una mirada integral con equipos multidisciplinares, orientada hacia la gestión eficaz del dolor y otros síntomas $(23,61)$.

Países como Europa, Canadá, Australia y Estados Unidos han logrado la integración del cuidado paliativo a la medicina convencional del sistema de salud, desarrollando en mayor medida niveles de atención comunitaria (domicilio, hospicio y consulta en centro comunitario) (8). En América Latina y el Caribe, Costa Rica y Chile son países que se caracterizan por el desarrollo de servicios multinivel para la población que requiere cuidado paliativo (44). Colombia se ubica en el grupo de países con provisión aislada de los servicios de cuidado paliativo (44).

El desarrollo y la promoción de los cuidados paliativos implica no sólo la construcción de políticas que reconozcan a la atención paliativa, sino que también se requieren sistemas de apoyo desde todos los niveles de atención, fortalecimiento de las acciones comunitarias, desarrollo de habilidades personales en los profesionales de la salud para la promoción del cuidado paliativo en las diferentes fases de la enfermedad y desde todas las dimensiones del ser humano, junto con la integración de los servicios de salud para lograr el acceso y cobertura universal (62). Además, se requiere por parte de los profesionales de la salud, la incorporación de los aspectos culturales en los procesos de atención como un elemento de mejora en los resultados de la salud (63).

\section{Conflicto de intereses}

El autor de este artículo no declara conflicto de interés. La fuente de financiación para el desarrollo fue con recursos propios.

\section{Referencias}

1. Montalvo-Prieto A, Cabrera-Nanclares B, QuiñonesArrieta S. Enfermedad crónica y sufrimiento: revisión de literatura. Aquichán. 2012;12(2):134-143.

2. Costa E, Fadel L. Cuidados paliativos no câncer e os princípios doutrinários do SUS. Saúde em Debate. 2015; 39(106):881-892.

3. De Lima L, Perez-Castells $M$, Berenguel $M$, Monti $C$, Aguilar A, Ahumada M, et al. Indicadores de Cuidado Paliativo ALCP. 1a edicion. Houston: IAHPC Press; 2013.

4. Orozco I, Ruelas L. Estudio exploratorio sobre las condiciones por una muerte digna: percepciones contradictorias. Psicología y Ciencia Social. 2009; 11(1):612.

5. Aparecida J, et al. Perfil dos pacientes com indicação de cuidados paliativos internados no Hospital Júlia Kubistchek - FHEMIG. Rev Med Minas Gerais. 2015; 25(1):25-29

6. Takauti D, Campos E. Los cuidados paliativos y la atención primaria de salud: scoping review. Rev. Bioét. 2015; 23(3):596-610.

7. Segura I. Percepción de la calidad de vida en personas con situación de enfermedad crónica en una Institución Prestadora de Servicios de Salud Barranquilla. [Tesis de maestría]. Bogotá: Editorial Facultad de enfermería Universidad Nacional de Colombia; 2012: 24-26

8. Hajjar R, Charalambous H, Baider L, Silbermann M. International palliative care: Middle East experience as a model for global palliative care. Clin Geriatr Med. 2015; 31(2):281-294.

9. Marcucci F, Cabrera M, Baquero A, Maroneze M, deBarros E, Martins V, et al. Identification and characteristics of patients with palliative care needs in Brazilian primary care. BMC Palliative Care. 2016; 15:51.

10. Vera W. Cuidado humano: La vulnerabilidad de ser enfermo y su dimensión de trascendencia. Index Enferm. 2014; 23(4): 234-238.

11. Getino M. Estudio cualitativo a pacientes de cáncer con soporte paliativo en atención hospitalaria. Ciência \& Saúde Coletiva. 2013; 18(9):2531-2539.

12. Barroso I, Grau J. Eutanasia y cuidados paliativos: ¿diferentes aristas de un mismo problema?. Psicología y Salud. 2012; 22(1):5-25.

13. Almeida CSL, Sales CA, Marcon SS. The existence of nursing in caring for terminally ills'life: a phenomenological study. Rev. esc. enferm. USP. 2014; 48(1):34-40.

14. Silva WCBP, et al. Nursing team perception of oncological palliative care: a phenomenological study. Online braz j nurs. 2014; 13(1):72-81.

15. Miranda M, Chagas M, Luzia J, Lorenzini A. Nursing work at night in palliative oncology care. Rev. Latino-Am. Enfermagem. 2013; 21(3):773-779.

16. De Araújo D, De Costa GF. Cuidados paliativos oncológicos: tendências da produção científica. R. Enferm. 2011; 1(2):238-245.

17. Buggey J, Mentz R, Galanos A. End-of-life Heart Failure Care in the United States. Heart Failure Clin. 2015; $11: 615-623$

18. Organización Mundial de la Salud. Control del Cáncer: aplicación de los conocimientos, Guía para desarrollar programas eficaces cuidados paliativos. Ed Traducida España; 2007; p. 1-50. [Fecha de consulta: Junio 29 del 
2015]. Disponible en: http://whqlibdoc.who.int/ publications/2007/9789243546995 spa.pdf

19. Siouta N, et al. Integrated palliative care in Europe: a qualitative systematic literature review of empirically-tested models in cancer and chronic disease. BMC Palliative Care. 2016; 15:51-56.

20. Clark D, Graham F, Centeno C. Changes in the world of palliative care. Medicine. 2015; 4(3):696-698.

21. Sarmiento-Medina MI. El cuidado paliativo: un recurso para la atención del paciente con enfermedad terminal. Revista Salud Bosque. 2011;1(2):23-37.

22. Tripodoro $\mathrm{V}$, et al. Atención paliativa en personas con enfermedades crónicas avanzadas. Medicina (Buenos Aires). 2016; 76:139-147.

23. Morrison S. Models of palliative care delivery in the United States. Curr Opin Support Palliat Care. 2013; 7(2): 201-206.

24. Pereira P, Faleiros D, Menossi MJ, García A. Cuidados paliativos para adolescentes com câncer: uma revisão da literature. Rev Bras Enferm, Brasília. 2009; 62(1):107-112.

25. Aguilera R, González J. La muerte como límite antropológico. El problema del sentido de la existencia humana. Gazeta de Antropología. 2009; 25(2):1-10.

26. Hodelín R. Evolución histórica y dilemas sociales de la muerte. Biblioteca Virtual de Salud. 2013; 17(17):1-3.

27. Rodriguez AM. El cuidador y el enfermo en el final de la vida - Familia y/o persona significativa. Enfermería Global. 2010; 18(1):1-9.

28. Dantas MM, Amazonas MC. The Illness Experience: Palliative Care Given the impossibility of Healing. Rev Esc Enferm USP. 2016; 50 Spec:47-53.

29. Gleick J. Caos: La creación de una ciencia. España: Crítica; 2012.

30. Costal M, Coelho J. Livre como uma borboleta: simbologia e cuidado paliativo. Rev. bras. geriatr. gerontol. 2015; 18(3):631-641.

31. Souza C. Ortotanásia, cuidados paliativos e direitos humanos. Rev Soc Bras Clin Med. 2015; 13(1):14-7.

32. Gherardi C. Suspensión y/o retiro del soporte vital. Buenos Aires: Universidad de Buenos Aires; 2014:255-270.

33. Álvarez C. Las diferentes concepciones de la muerte en las principales culturas de la humanidad: origen de su significación ante la sociedad actual. Buenos Aires: Universidad de Cartapacio Argentina; 2002:10-40.

34. Povedano-Jiménez M, Catalán-Matamoros D, GranadosGámez G. La comunicación de los profesionales sanitarios en Cuidados Paliativos. Rev Esp Comun Salud. 2014; 5(2):78-91.

35. Dzingina M, Higginson I. Public Health and Palliative Care in 2015. Clin Geriatr Med. 2015; 1:253-263.

36. Organización Mundial de la Salud. Global Atlas of Palliative Care at the End of Life. 2014. [Fecha de consulta: Mayo 5 del 2015]. Disponible en: http://www.who.int/nmh/ Global_Atlas_of_Palliative_Care.pdf .

37. Hermansa K, Spruyttea $\bar{N}$, Cohenb J, Audenhovea C, Declercq A. Usefulness, feasibility and face validity of the interRAl Palliative Care instrument according to care professionals in nursing homes: A qualitative study. International Journal of Nursing Studies. 2016; 62:90-99

38. Fernandes R, De Oliveira S, Oliveira M, Barbosa K, Medeiros R. Cuidados paliativos: desafios para cuidadores e profissionais de saúde. Revista de Psicologia. 2015; 27(2):165-176

39. Organización Mundial de la salud. Fortalecimiento de los cuidados paliativos como parte del tratamiento integral a lo largo de la vida. 2014; WHA67.19: 1-6.

40. Bruera E, Wenk R, de Lima L. Palliative Care in Developing Countries: Principles and Practice. Houston: IAHCP Press, 2004.

41. Patel K, Masi D. Palliative Care in the Era of Health Care Reform. Clin Geriatr Med. 2015; 1:265-270.

42. West $\mathrm{E}$, et al. Hospice care in the Netherlands: who applies and who is admitted to inpatient care?. BMC Health Services Research. 2016; 16(33):1-8.

43. Coelho A, Parola V, Escobar-Bravo M, Apóstolo A. Comfort experience in palliative care: a phenomenological study. BMC Palliative Care. 2016; 15(71):1-8.

44. Pastrana T, De Lima L, Pons JJ, Centeno C. Atlas de Cuidados Paliativos de Latinoamérica. Houston: IAHPC Press, 2013.

45. Slomka J, Prince-Paul M, Webel A, Daly B. Palliative Care, Hospice, and Advance Care Planning: Views of People Living with HIV and Other Chronic Conditions. Journal of the association of nurses in aids care. 2016; 27(4):476-484.

46. Parodi J, Morante R, Hidalgo L, Carreño R. Propuesta de políticas sobre cuidados paliativos para personas adultas mayores en Latinoamérica y el Caribe. Horiz Med. 2016; 16(1):69-74.

47. Annavarapu S, Marupaka J, Kumar N. Impact of teaching session on concepts of palliative care in medical undergraduates. International Journal of Basic \& Clinical Pharmacology. 2016; 5(1):188-191.

48. Díaz VE, Ruíz MA. La experiencia de morir: Reflexiones sobre el duelo anticipado. Facultad de Ciencias Humanas. Revista de Psicoanálisis. 2011; 11:163-178.

49. Saunders C. Vida y muerte digna: cuidados paliativos. Arch Neurocien. 2013; 18(3): 111-112.

50. Garrido MA, Gil XB. Caracterización de las necesidades psicosociales del enfermo oncológico terminal. Humanidades Médicas. 2012; 12(2):203-216.

51. Grupo de Trabalho em Cuidados Paliativos do Cremesp. Cuidado Paliativo. São Paulo: Conselho Regional de Medicina do Estado de São Paulo (Cremesp), 2008.

52. Espinoza M, Sanhueza O. Factores relacionados a la calidad del proceso de morir en la persona con cáncer. Rev. Latino-Am Enfermagem. 2010; 18(4):1-8.

53. Banco mundial. Gasto en salud, total (\% del PIB). 2016. Fecha de publicación [2016; 27/08/2016. Disponible en: http://datos.bancomundial.org/indicador/SH.XPD.TOTL.Z S.

54. Colombia. Ley $N^{\circ} 1733$ DE 2014. 2014. Fecha de publicación [2014; 27/08/2016. Disponible en: http://wsp. presidencia.gov.co/ Normativa/Leyes/Documents/LEY\% 201733\%20DEL\%2008\%20DE\%20SEPTIEMBRE\%20 DE\%202014.pdf.

55. Teresi J. Measurement equivalence of the Patient Reported Outcomes Measurement Information Pain Interference short form items: Application to ethnically diverse cancer and palliative care populations. Psychological Test and Assessment Modeling. 2016; 58(2):309-352.

56. León M, Florez S, De Lima L, Ryan K. Integrating palliative care in public health: The Colombian experience following and International Pain Policy Fellowship. Palliat Med. 2011; 25(4):365-9.

57. Bond C, Lavy V, Wooldridge R. Juego de herramientas para el Cuidado Paliativo: Mejorando el cuidado desde la raíz en sectores limitados en recursos. Buenos Aires: Gráfica Sur Editora SRL, 2008.

58. Celedón C. Sufrimiento y muerte en un paciente terminal. Rev. Otorrinolaringol. Cir. Cabeza Cuello. 2012; 72:261266

59. Teruel J, Rexach L, Burguera V, Gomis A, FernandezLucas M, Rivera M, et al. Home Palliative Care for Patients with Advanced Chronic Kidney Disease: Preliminary Results. Healthcare. 2015; 3:1064-1074.

60. Miranda M, Büscher A, Chagas M, Machado S. Visiting hospices in Germany and United Kingdom from the perspective of palliative care. Esc Anna Nery 2015; 19(2):369-375

61. Van den Block L, Smets T, van Dop N, Adang E, Andreasen $\mathrm{P}$, Collingridge Moore D, et al. Comparing Palliative Care in Care Homes Across Europe (PACE): Protocol of a Crosssectional Study of Deceased Residents in 6 EU Countries. J Am Med Dir Assoc. 2016; 17(6): 566.e1-7.

62. Archibald D, Patterson R, Haraldsdottir E, Hazelwood M Fife S, Murray S. Mapping the progress and impacts of public health approaches to palliative care: a scoping review protocol. BMJ Open 2016; 6(7): e012058.

63. López $L$. La competencia cultural, una clave para mejores resultados en salud.Revista MedUNAB 2016;18(3):162-3. 